\title{
THE WAKE OF A FINITE ROTATING DISC
}

\author{
L. M. LESLIE
}

(Received 10 November 1969; revised 11 February 1970)

Communicated by A. F. Pillow

\section{Introduction}

When a disc rotates in a fluid at rest, fluid near the disc acquires azimuthal momentum because of the viscous torque of the disc and outwards radial momentum under the action of centrifugal forces. The resultant flow is essentially a swirling jet. Away from the disc continuity requires the existence of an axial flow towards the disc to compensate for the fluid which has been thrown outwards. If the disc is finite there is a discontinuity in the boundary conditions at the edge of the disc where the no-slip condition is suddenly replaced by a condition of zero stress in the plane of the disc. The flow discharged from the edge of the disc is essentially a wake embedded in a swirling radial jet. It appears that no investigation of this wake has yet been made.

Before proceeding, the results of research into a closely related flow problem, namely, the wake that forms downstream from the trailing edge of a flat plate placed parallel to a uniform stream, should be examined. The boundary layer equations for the flow near a semi-infinite flat plate were first solved by Blasius [1]. Goldstein [3] went one step further by solving the boundary layer equations for the wake that develops downstream from the trailing edge of a finite flat plate. Goldstein assumed that the boundary layer approximation is valid downstream from the trailing edge itself and obtained a solution for the near wake, the near wake being defined by $0<x \ll l$, where $x$ denotes distance downstream from the trailing edge and $l$ is the length of the plate. However, as has been shown by Stewartson [6], [7], the boundary layer approximation fails in the neighbourhood of the trailing edge and the full Navier-Stokes must be used there. Goldstein's solution is therefore not appropriate to all of the near wake but to a slightly smaller domain which Stewartson refers to as the Goldstein wake. Stewartson [7] has demonstrated that for high Reynolds number flows the neighbourhood of the trailing edge, which connects the Blasius flow over the plate with the Goldstein wake, may be split into two main regions. Suppose that the Reynolds number of the flow is defined by $\operatorname{Re}=U_{0} l / v$, where $U_{0}$ is the free stream velocity and $v$ is the kinematic viscosity; that $\varepsilon$ is defined by $\varepsilon^{8}=\operatorname{Re}^{-1}$; and that $y$ measures 
perpendicular distance from the $x$ axis. Then Stewartson has shown that the neighbourhood of the trailing edge consists of 'a central region in which $x \sim \varepsilon^{6} l$, $\mathrm{y} \sim \varepsilon^{6} l \ldots$ and an intermediate region in which $x \sim \varepsilon^{3} l, y \sim \varepsilon^{3} l$.

The primary aim of this paper is to show how the method developed by Goldstein for the flat plate may be extended to obtain a solution for the near wake of the discharge from a finite rotating disc. As in the case of the flat plate, this solution is not valid in the neighbourhood of the edge of the disc. Any examination of the flow in this neighbourhood would necessarily involve the full Navier-Stokes equations and no such analysis will be attempted here. Following Stewartson, the domain for which Goldstein's solution is appropriate will be referred to as the Goldstein wake of a finite rotating disc. Smith [5] has extended Goldstein's method to advance the solution for the free convection boundary layer along a vertical plate past the level at which a discontinuity in plate temperature occurs. Smith has included a detailed discussion of the method and only a brief outline is necessary here. The method, as originally used by Goldstein, consists in forming two expansions for the stream function, one valid near the wake axis (the inner expansion) and the other valid away from the wake axis (the outer expansion). The inner expansion satisfies the boundary conditions at

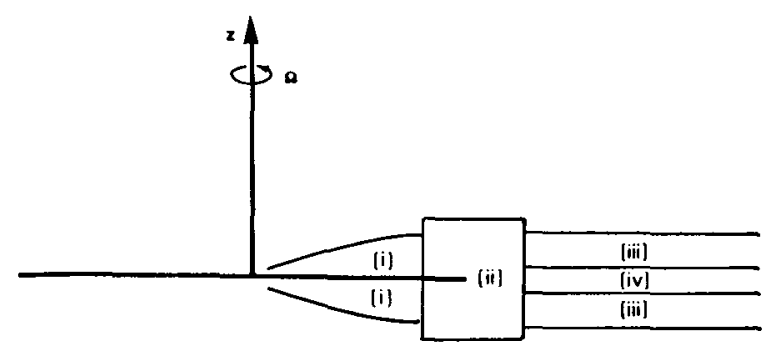

Figure (1a)

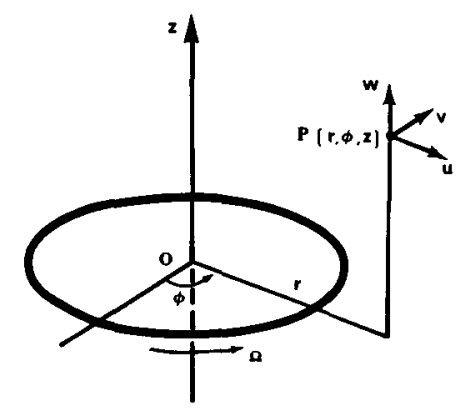

Figure (1b)

Fig. 1(a): Illustrates the various flow regions near the edge of the disc: in (i) Cochran's (1934) solution is valid; in (ii) the boundary layer equations are not valid; while (iii) and (iv) are the Goldstein outer and inner wake regions respectively. (b) Shows the co-ordinate system used. 
the wake axis and its form is suggested by the Blasius solution. The outer expansion satisfies the constraints imposed on the solution far from the wake axis and the two expansions are matched by re-arranging the asymptotic expression for the inner expansion into the same form as the outer expansion. For the rotating disc Goldstein's method is extended by forming expansions not only of the stream function but also of the azimuthal velocity, and the form of the inner expansions is derived from Cochran's [2] improvement on the original solution by von Karman [8] for the flow near an infinite rotating disc. The main flow regions near the edge of the disc are illustrated in Fig. 1(a).

\section{The equations of motion}

Let $(r, \varphi, z)$ be cylindrical polar co-ordinates with origin at the centre of the disc and $z$ axis of rotation. The velocity vector is $(u, v, w)$ and the finite disc is of radius $a$.

The boundary layer equations for flow in the Goldstein wake near a rotating disc are, as given by Schlichting [4]:

$$
\begin{gathered}
u \frac{\partial u}{\partial r}+w \frac{\partial u}{\partial z}-\frac{v^{2}}{r}=v \frac{\partial^{2} u}{\partial z^{2}}, \\
u \frac{\partial v}{\partial r}+w \frac{\partial v}{\partial z}+\frac{u v}{r}=v \frac{\partial^{2} v}{\partial z^{2}} \\
w \frac{\partial w}{\partial z}=-\frac{1}{\rho} \frac{\partial p}{\partial z}+v \frac{\partial^{2} w}{\partial z^{2}}, \\
\frac{\partial}{\partial r}(r u)+\frac{\partial}{\partial z}(r w)=0
\end{gathered}
$$

Since the boundary layer equations involve the assumption that $p$ and $w$ are independent of $r$ (2.3) may be written as

$$
\frac{p}{\rho}+\frac{1}{2} w^{2}-v \frac{\partial w}{\partial z}=\text { const. }
$$

It remains only to solve (2.1), (2.2), (2.4) subject to the boundary conditions of the problem. Then $p$ may be calculated from (2.5).

For an infinite plate rotating in a still environment the boundary conditions are

$$
\begin{aligned}
& z=0: \quad u=w=0, \quad v=r \Omega ; \\
& z=\infty: \quad u=v=0, \quad w \text { finite; }
\end{aligned}
$$

where $\Omega$ is the angular velocity of the disc. Equations (2.1), (2.2), (2.4), (2.6) have been solved by von Kármán [8] and Cochran [2]. They defined new variables $F, G, H, Z$, by 


$$
\begin{aligned}
u & =r \Omega F(Z), \\
v & =r \Omega G(Z), \\
w & =\sqrt{v \Omega} H(Z), \\
z & =\sqrt{\frac{v}{\Omega}} Z .
\end{aligned}
$$

The functions $F, G, H$ satisfy the following simultaneous system of ordinary differential equations

$$
\begin{aligned}
F^{2}+F^{\prime} H-G^{2}-F^{\prime \prime} & =0 \\
2 F G+H G^{\prime}-G^{\prime \prime} & =0, \\
2 F+H^{\prime} & =0,
\end{aligned}
$$

together with the transformed boundary conditions

$$
\begin{array}{rll}
Z=0: & F=H=0, & G=1 ; \\
Z=\infty: & F=G=0, & H \text { finite. }
\end{array}
$$

The functions $F, G, H, F^{\prime}, G^{\prime}$ have been tabulated by Cochran.

\section{The continuation problem}

The continuation problem is to advance the solution to equations (2.1), (2.2), (2.4) radially outwards, given the Cochran velocity profiles at the edge of the disc. A transformation to non-dimensional variables of form appropriate to the outflow from the disc edge is

$$
\begin{aligned}
u & =a \Omega U, \\
v & =a \Omega V, \\
w & =\sqrt{v \Omega} W, \\
r & =a(1+R), \\
z & =\sqrt{\frac{v}{\Omega}} Z .
\end{aligned}
$$

The transformed equations are

$$
\begin{gathered}
U \frac{\partial U}{\partial R}+W \frac{\partial U}{\partial Z}-\frac{V^{2}}{1+R}=\frac{\hat{\sigma}^{2} U}{\partial Z^{2}}, \\
U \frac{\partial V}{\partial R}+W \frac{\partial V}{\partial Z}+\frac{U V}{1+R}=\frac{\partial^{2} V}{\partial Z^{2}}, \\
\frac{\partial}{\partial R}\{(1+R) U\}+\frac{\partial}{\partial Z}\{(1+R) W\}=0 .
\end{gathered}
$$


The profiles of $U, V, W$ at $R=0$, which are the Cochran profiles $F, G, H$, may be expanded in Maclaurin series

$$
\begin{aligned}
& U=a_{1} Z+a_{2} Z^{2}+a_{3} Z^{3}+\cdots, \\
& V=b_{0}+b_{1} Z+b_{2} Z^{2}+\cdots,
\end{aligned}
$$

where, by successive differentiation of equations (2.7) it is easily shown that

$$
\begin{array}{ll}
a_{1}=0.510, & b_{1}=-0.616 ; \\
a_{2}=-1 / 2 !, & b_{2}=0 ; \\
a_{3}=-2 b_{1} / 3 !, & b_{3}=2 a_{1} / 3 !
\end{array}
$$

\section{The inner solution}

From the third of equations (3.1) there is a stream function $\psi$ such that

$$
\begin{aligned}
& U=\frac{1}{1+R} \frac{\partial \psi}{\partial Z}, \\
& W=-\frac{1}{1+R} \frac{\partial \psi}{\partial R} .
\end{aligned}
$$

Following Goldstein, new independent variables $\xi$ and $\eta$ are defined by

$$
\begin{aligned}
& \xi=R^{\frac{1}{3}}, \\
& \eta=Z R^{-\frac{1}{3}} / 3,
\end{aligned}
$$

and the stream function and azimuthal velocity are taken to be

$$
\begin{aligned}
\psi & =\xi^{2} f(\xi, \eta) \\
V & =g(\xi, \eta) .
\end{aligned}
$$

Then, from (4.1) and (4.3)

$$
\begin{aligned}
U & =\frac{\xi}{3\left(1+\xi^{3}\right)} f_{\eta}, \\
W & =-\frac{\xi^{-1}}{3\left(1+\xi^{3}\right)}\left(2 f+\xi f_{\xi}-\eta f_{\eta}\right),
\end{aligned}
$$

and the initial conditions (3.2) become

$$
\begin{aligned}
& U=a_{1}(3 \eta \xi)+a_{2}(3 \eta \xi)^{2}+\cdots \\
& V=b_{0}+b_{1}(3 \eta \xi)+b_{2}(3 \eta \xi)^{2}+\cdots
\end{aligned}
$$

A comparison of (4.4) and (4.5) suggests the following power series expansions in $\xi$ for $f$ and $g$ : 


$$
\begin{aligned}
& f=\sum_{m=0}^{\infty} h_{m}(\eta) \xi^{m} \\
& g=\sum_{m=0}^{\infty} k_{m}(\eta) \xi^{m}
\end{aligned}
$$

If these series are substituted in equations (4.4) and (3.1), two sets of ordinary differential equations are obtained by equating powers of $\xi$ to zero. The first four equations of each set are:

$$
\begin{aligned}
& h_{0}^{\prime \prime \prime}+2 h_{0} h_{0}^{\prime \prime}-h_{0}^{\prime 2}=0 \\
& h_{1}^{\prime \prime \prime}+2 h_{0} h_{1}^{\prime \prime}-3 h_{0}^{\prime} h_{1}^{\prime}+3 h_{0}^{\prime \prime} h_{1}=-27 k_{0}^{2}, \\
& h_{2}^{\prime \prime \prime}+2 h_{0} h_{2}^{\prime \prime}-4 h_{0}^{\prime} h_{2}^{\prime}+4 h_{0}^{\prime \prime} h_{2}=2 h_{1}^{\prime 2}-3 h_{1} h_{1}^{\prime \prime}-54 k_{0} k_{1}, \\
& h_{3}^{\prime \prime \prime}+2 h_{0} h_{3}^{\prime \prime}-5 h_{0}^{\prime} h_{3}^{\prime}+5 h_{0}^{\prime \prime} h_{3}=5 h_{1}^{\prime} h_{2}^{\prime}-3 h_{1} h_{2}^{\prime \prime}-4 h_{1}^{\prime \prime} h_{2} \\
&-27 k_{1}^{2}-54 k_{0} k_{2}-4 h_{0}^{\prime 2}+2 h_{0} h_{0}^{\prime \prime}
\end{aligned}
$$

and

$$
\begin{gathered}
k_{0}^{\prime \prime}+2 h_{0} h_{0}^{\prime}=0, \\
k_{1}^{\prime \prime}+2 h_{0} k_{1}^{\prime}-h_{0}^{\prime} k_{1}=-3 h_{1} k_{0}^{\prime}, \\
k_{2}^{\prime \prime}+2 h_{0} k_{2}^{\prime}-2 h_{0}^{\prime} k_{2}=h_{1}^{\prime} k_{1}-3 h_{1} k_{1}^{\prime}-4 h_{2} k_{0}^{\prime}, \\
k_{3}^{\prime \prime}+2 h_{0} k_{3}^{\prime}-3 h_{0}^{\prime} k_{3}=3 h_{0}^{\prime} k_{0}+2 h_{1}^{\prime} k_{2}+h_{2}^{\prime} k_{1}-3 h_{1} k_{2}^{\prime}-4 h_{2} k_{1}^{\prime}-5 h_{3} k_{0}^{\prime} .
\end{gathered}
$$

The boundary conditions to be satisfied by $U, V$ and $W$ in the region $R>0$ are

$$
\begin{aligned}
& Z=0: W=\frac{\partial U}{\partial Z}=\frac{\partial V}{\partial Z}=0 \\
& Z=\infty: U=V=0, W \text { finite. }
\end{aligned}
$$

The boundary conditions at $Z=0$ transform to

$$
\eta=0: h_{m}=h_{m}^{\prime \prime}=k_{m}^{\prime}=0,
$$

while the boundary conditions as $\eta \rightarrow \infty$ are obtained by equating the series expansions of $U$ and $V$ with the initial conditions in the form (4.5). Thus

$$
\begin{aligned}
& \operatorname{Lim}_{\eta \rightarrow \infty} \frac{h_{0}^{\prime}}{\eta}=3^{2} a_{1}, \\
& \operatorname{Lim}_{\eta \rightarrow \infty} \frac{h_{1}^{\prime}}{\eta^{2}}=3^{3} a_{2}, \\
& \operatorname{Lim}_{\eta \rightarrow \infty} \frac{h_{2}^{\prime}}{\eta^{3}}=3^{4} a_{3}, \\
& \operatorname{Lim}_{\eta \rightarrow \infty} \frac{h_{3}^{\prime}-h_{0}^{\prime}}{\eta^{4}}=3^{5} a_{4} ;
\end{aligned}
$$




$$
\operatorname{Lim}_{\eta \rightarrow \infty} \frac{k_{m}}{\eta^{m}}=3^{m} b_{m} \quad(m=0,1,2,3) .
$$

The solution for $h_{0}$

The equation for $h_{0}$ was investigated by Goldstein op. cit. He obtained the following solution

$$
h_{0}=\beta_{0} \eta+\beta_{0}^{2} \frac{\eta^{3}}{3 !}-2 \beta_{0}^{3} \frac{n^{5}}{5 !}+10 \beta_{0}^{4} \frac{\eta^{7}}{7 !} \cdots,
$$

which is a MacLaurin series whose coefficients are computed by successive differentiations of equation (4.7) for $f_{0}$. Let $H_{0}(\eta)$ be the solution to equation (4.7) for $h_{0}$ with $\beta_{0}=1$. This solution is related to $h_{0}$ by

$$
h_{0}(\eta)=\beta_{0}^{\frac{1}{1}} H_{0}\left(\beta_{0}^{\frac{1}{2}} \eta\right) \text {. }
$$

Therefore

From the first of equations (4.10a)

$$
\begin{aligned}
\operatorname{Lim}_{\eta \rightarrow \infty} \frac{h_{0}^{\prime}(\eta)}{\eta} & =\beta_{0}^{\frac{3}{3}} \operatorname{Lim}_{\eta \rightarrow \infty} \frac{H_{0}^{\prime}(\eta)}{\eta}, \\
& =\beta_{0}^{\frac{3}{2}} \operatorname{Lim}_{\eta \rightarrow \infty} H_{0}^{\prime \prime}(\eta) .
\end{aligned}
$$

which implies

$$
\begin{aligned}
\operatorname{Lim}_{\eta \rightarrow \infty} \frac{h_{0}^{\prime}(\eta)}{\eta} & =3^{2} a_{1}, \\
& =\alpha_{0},
\end{aligned}
$$

$$
\beta_{0}=\left\{\alpha_{0} / \operatorname{Lim}_{\eta \rightarrow \infty} H_{0}^{\prime \prime}(\eta)\right\}^{\frac{2}{3}}
$$

The asymptotic behaviour of $h_{0}$ as $n \rightarrow \infty$ is, according to Goldstein

$$
h_{0} \sim \frac{1}{2} \alpha_{0} \eta^{\prime 2}
$$

where $n^{\prime}=\eta+\delta_{0}, \delta_{0}$ being an arbitrary constant. This may be written as

$$
h_{0} \sim A_{0} \eta^{2}+B_{0} \eta+C_{0} .
$$

$h_{0}(\eta)$ is the solution to equation (4.7) for $h_{0}$ with $H_{0}^{\prime}(0)=1, H_{0}(0)=H_{0}^{\prime \prime}(0)=0$. Thus $H_{0}(\eta), H_{0}^{\prime}(\eta)$ and $H_{0}^{\prime \prime}(\eta)$ may be obtained numerically using a RungeKutta technique and it was found that for large values of $\eta$

$$
H_{0}(\eta) \sim 0.42357(\eta+0.65364)^{2} .
$$

From (4.12) and (4.14) the values of $\beta_{0}$ and $\delta_{0}$ may be found. They are

$$
\begin{aligned}
& \beta_{0}=3.0849, \\
& \delta_{0}=0.37215 .
\end{aligned}
$$

Equation (4.7) for $h_{0}$ may now be solved, using $\beta_{0}$ as the initial value of $h_{0}^{\prime}$. 
The solution for $k_{0}$

The solution to equation (4.8) for $k_{0}$, satisfying boundary conditions (4.9) and $(4.10 \mathrm{~b})$ is a constant,

$$
k_{0}=b_{0} .
$$

The solution for $h_{1}$

Let $H_{1 p}(\eta)$ be a particular integral of the equation (4.7) for $h_{1}$ and $H_{1 c}(\eta)$ the complementary function for which $H_{1 c}(0)=H_{1 c}^{\prime \prime}(0)=0$ and $H_{1 c}^{\prime \prime}(0)=1$. Then

$$
H_{1 c}(\eta)=\eta+3 \beta_{0} \frac{n^{3}}{3 !}-6 \beta_{0}^{2} \frac{\eta^{5}}{5 !}+\cdots
$$

and, choosing a particular integral such that $H_{1 p}(0)=H_{1 p}^{\prime}(0)=H_{1 p}^{\prime \prime}(0)=0$,

$$
H_{1 p}(\eta)=-27 b_{0}^{2}\left(\frac{y^{3}}{3 !}-\beta_{0} \frac{y^{5}}{5 !}+\cdots\right)
$$

The solution for $h_{1}$ is therefore

$$
h_{1}(\eta)=H_{1 p}(\eta)+\lambda_{1} H_{1 c}(\eta),
$$

where $\lambda_{1}$ is to be determined from (4.10a). Asymptotically, the equation for $f_{1}$ is satisfied by

$$
A_{1} \eta^{\prime 3}+\bar{C}_{1} \eta^{\prime}-\left(9 b_{0}^{2}+A_{1}\right) \alpha_{0}^{-1},
$$

where exponentially small terms have been neglected. From (4.14a)

$$
A_{1}=3^{2} a_{2} \text {. }
$$

Consequently the following asymptotic equality defines $\lambda_{1}$ :

$$
\lambda_{1} \cong\left(3 A_{1} \eta^{\prime}-H_{1 p}^{\prime \prime}(\eta)\right) / H_{1 c}^{\prime \prime}(\eta) .
$$

The functions $H_{1 p}(\eta), H_{1 c}(\eta)$ and their first two derivatives were tabulated by numerical solution of the homogeneous and non-homogeneous equations respectively. The value of $\lambda_{1}$ was then found by substituting $H_{1 p}^{\prime \prime}(\eta)$ and $H_{1 c}^{\prime \prime}$ into (4.19), for "sufficiently" large $\eta$ ( $\eta=7$ was found to be large enough to make the exponential terms negligible). The value of $\bar{C}_{1}$ was then found by comparing the derivative of (4.17) with the right-hand side of $(4.1 \mathrm{~b})$ for "sufficiently" large $\eta$.

If (4.17) is expressed as a polynomial in $\eta$, it is noticed that the asymptotic behaviour of $h_{1}$ is

$$
h_{1} \sim A_{1} \eta^{3}+B_{1} \eta^{2}+C_{1} \eta+D_{1},
$$

where $A_{1}$ is given by (4.18) and $B_{1}, C_{1}$, and $D_{1}$ are related to $A_{1}$ and $\bar{C}_{1}$ by 


$$
\begin{aligned}
& B_{1}=3 A_{1} \delta_{0} \\
& \vec{C}_{1}=C_{1}+3 A_{1} \delta_{0}^{2} \\
& D_{1}=A_{1} \delta_{0}^{3}+C_{1} \delta_{0}-\left(9 b_{0}^{2}+A_{1}\right) \alpha_{0}^{-1} .
\end{aligned}
$$

The solution for $k_{1}$

The equation (4.8) for $k_{1}$ is homogeneous since $k_{0}$ is constant. If $K_{1 c}$ is the complementary function satisfying $K_{1 c}(0)=1, k_{1 c}^{\prime}(0)=0$, then

$$
k_{1}=\mu_{1} k_{1 c},
$$

where $\mu_{1}$ is to be determined from (4.10b). The asymptotic behaviour of $k_{1}$ is easily shown to be

$$
\begin{aligned}
k_{1} & \sim G_{1} \eta^{\prime} \\
& \sim G_{1} \eta+H_{1},
\end{aligned}
$$

where $H_{1}=\delta_{0} G_{1}$. From (4.10b)

$$
G_{1}=3 b_{1},
$$

hence, using (4.21), (4.22), (4.23)

$$
\mu_{1} \cong 3 b_{1} / k_{1 c}^{\prime} \text {. }
$$

The functions $k_{1 c}(\eta)$ and $k_{1 c}^{\prime}(\eta)$ were tabulated by numerical solution of the differential equation and the limiting value of the right-hand side of (4.23) was obtained.

The solutions for $h_{n}$ and $k_{n}(n=2,3)$

The solutions for $f_{n}$ and $g_{n}$ are:

$$
\begin{aligned}
& h_{2}(\eta)=H_{2 p}(\eta)+\lambda_{2} H_{2 c}(\eta), \\
& h_{3}(\eta)=H_{3 p}(\eta)+\lambda_{3} H_{3 c}(\eta),
\end{aligned}
$$

and

$$
\begin{aligned}
& k_{2}(\eta)=K_{2 p}(\eta)+\mu_{2} K_{2 c}(\eta), \\
& k_{3}(\eta)=K_{3 p}(\eta)+\mu_{3} K_{3 c}(\eta),
\end{aligned}
$$

where $H_{n p}, K_{n p}(n=2,3)$ are particular integrals and $H_{n c}, K_{n c}$ are complementary functions for the appropriate differential equations of (4.7) and (4.8). The functions $H_{n p}, H_{n c}, K_{n c}$ satisfy the same conditions at $\eta=0$ as $H_{1 p}, H_{1 c}, k_{1 c}$ respectively, while $K_{n p}(0)=K_{n p}^{\prime}(0)=0$. The $\lambda_{n}$ and $\mu_{n}$ are to be determined from (4.10a) and (4.10b) respectively, in the same manner that $\lambda_{1}$ and $\mu_{1}$ were determined.

The asymptotic forms of $h_{n}$ and $k_{n}$ may be inferred directly from the results of Smith op. cit. for his free-convection boundary layer problem. Thus $h_{n}$ and $k_{n}$ have the following asymptotic behaviour: 


$$
\begin{aligned}
& h_{2} \sim A_{2} \eta^{4}+B_{2} \eta^{3}+C_{2} \eta^{2}+D_{2} \eta+E_{2}, \\
& h_{3} \sim A_{3} \eta^{5}+B_{3} \eta^{4}+C_{3} \eta^{3}+D_{3} \eta^{2}+E_{3} \eta+F_{3},
\end{aligned}
$$

and

$$
\begin{aligned}
k_{2} & \sim G_{2} \eta^{2}+H_{2} \eta+I_{2}, \\
k_{3} & \sim G_{3} \eta^{3}+H_{3} \eta^{2}+I_{3} \eta+J_{3},
\end{aligned}
$$

where the coefficients $A_{2}, A_{3}$, etc., are obtained numerically.

\section{The outer solution}

The outer expansion is obtained as follows. The inner expansion for large $\eta$ is written in terms of the outer variable $Z$ and, with the assumption that re-arrangement is possible, equations (4.13), (4.15), (4.20), (4.22) and (4.27) combine to give

$$
\begin{aligned}
\psi \sim & A_{0}(Z / 3)^{2}+A_{1}(Z / 3)^{2}+\cdots \\
& +\xi\left(B_{0}(Z / 3)+B_{1}(Z / 3)^{2}+\cdots\right) \\
& +\xi^{2}\left(C_{0}+C_{1}(Z / 3)+C_{2}(Z / 3)^{2}+\cdots\right) \\
& +\xi^{3}\left(D_{1}+D_{2}(Z / 3)+\cdots\right) \\
& +\cdots
\end{aligned}
$$

and

$$
\begin{aligned}
V \sim & k_{0}+G(Z / 3)+G_{2}(Z / 3)^{2}+\cdots \\
& +\xi\left(H_{1}+H_{2}(Z / 3)+\cdots\right) \\
& +\xi^{2}\left(I_{2}+I_{3}(Z / 3)+\cdots\right) \\
& +\cdots
\end{aligned}
$$

The expressions (5.1) and (5.2) suggest the following expansions for the outer solution, provided $Z R^{-\frac{1}{5}}$ is sufficiently large:

$$
\begin{aligned}
& \psi=\psi_{0}(Z)+\frac{\xi}{1 !} \psi_{1}(Z)+\frac{\xi^{2}}{2 !} \psi_{2}(Z)+\cdots \\
& V=V_{0}(Z)+\frac{\xi}{1 !} V_{1}(Z)+\frac{\xi^{2}}{2 !} V_{2}(Z)+\cdots
\end{aligned}
$$

where

and

$$
\begin{aligned}
& \psi_{0}^{\prime}=a_{1} Z+a_{2} Z^{2}+a_{3} Z^{3}+\cdots \\
& \psi_{1}=\frac{1}{3} B_{0} Z+\frac{1}{9} B_{1} Z^{2}+\frac{1}{27} B_{2} Z^{3}+\cdots, \\
& \psi_{2}=2 C_{0}+\frac{2}{3} C_{1} Z+\frac{2}{9} C_{2} Z^{2}+\cdots \\
& \psi_{3}=6 D_{1}+2 D_{2} Z+\frac{2}{3} D_{3} Z^{2}+\cdots,
\end{aligned}
$$




$$
\begin{aligned}
V_{0} & =b_{0}+b_{1} Z+b_{2} Z^{2}+b_{3} Z^{3}+\cdots, \\
V_{1} & =H_{1}+\frac{1}{3} H_{2} Z+\frac{1}{9} H_{3} Z^{2}+\cdots \\
V_{2} & =I_{2}+\frac{1}{3} I_{3} Z+\cdots \\
V_{3} & =J_{3}+\cdots
\end{aligned}
$$

When the series (5.3) are substituted into the first two equations of (3.1), the following equations for $\psi_{n}$ and $V_{n}(n=1,2,3)$ are obtained:

$$
\begin{aligned}
& \psi_{1}^{\prime} \psi_{0}^{\prime}-\psi_{1} \psi_{0}^{\prime \prime}=0, \\
& \psi_{2}^{\prime} \psi_{0}^{\prime}-\psi_{2} \psi_{0}^{\prime \prime}=\psi_{1} \psi_{1}^{\prime \prime}-\psi_{1}^{\prime 2}, \\
& \psi_{3}^{\prime} \psi_{0}^{\prime}-\psi_{3} \psi_{0}^{\prime \prime}=6\left(\psi_{0}^{\prime \prime \prime}+V_{0}^{2}\right)+\psi_{1} \psi_{2}^{\prime \prime}-3 \psi_{1}^{\prime} \psi_{2}^{\prime}+2 \psi_{1}^{\prime \prime} \psi_{2},
\end{aligned}
$$

and

$$
\begin{aligned}
V_{1} \psi_{0}^{\prime}-V_{0}^{\prime} \psi_{1}= & 0 \\
V_{2} \psi_{0}^{\prime}-V_{0}^{\prime} \psi_{2}= & \psi_{1} V_{1}^{\prime}-\psi_{1}^{\prime} V_{1}, \\
V_{3} \psi_{0}^{\prime}-V_{0}^{\prime} \psi_{3}= & 6\left(V_{0}^{\prime \prime \prime}+V_{0} \psi_{0}^{\prime}\right)+2 \psi_{2} V_{1}^{\prime}+\psi_{1} V_{2}^{\prime} \\
& -2 \psi_{1}^{\prime} V_{2}-\psi_{2}^{\prime} V_{1} .
\end{aligned}
$$

The first equation of (5.6) may be integrated immediately to yield

$$
\psi_{1}=k \psi_{0}^{\prime}
$$

the constant $k$ being found from a comparison of the first two expansions of (5.4). Hence

$$
k=B_{0} / 3 a_{1} .
$$

The expansion for $\psi_{2}$ may now be integrated to give

$$
\psi_{2}=k^{2} \psi_{0}^{\prime \prime}+l \psi_{0}^{\prime},
$$

with the first and third expansions of (5.4) implying that

The solution for $\psi_{3}$ is

$$
l=2\left(C_{1}-3 a_{2} k^{2}\right) / 3 a_{1} .
$$

$$
\psi_{3}=6 \psi_{0}^{\prime}\left(\int_{0}^{z} \frac{V_{0}+\psi_{0}^{\prime \prime \prime}}{\psi_{0}^{\prime 2}} d Z+m\right)+k^{3} \psi_{0}^{\prime \prime \prime}+3 k l \psi_{0}^{\prime \prime} .
$$

The coefficient of $\psi_{0}^{\prime}$ in the expression for $\psi_{3}$ involves an arbitrary constant of integration, $m$. The expansion for $\psi_{3}$ in powers of $y$ is

$$
\Psi_{3}=k\left(3 a_{2} k^{2}-3 a_{1} l\right)+6\left(a_{1} m+a_{3} k^{3}+a_{2} k l\right) y+0\left(y^{2}\right),
$$

and by comparison with the expansion (5.4) for $\Psi_{3}$

$$
m=\left(\frac{1}{3} D_{2}-a_{3} k^{2}-a_{2} k l\right) / a_{1} .
$$


The functions $V_{n}(n=1,2,3)$ are now defined in terms of $k, l, m$ and the initial solutions $\Psi_{0}$ and $V_{0}$. They are:

$$
\begin{aligned}
V_{1}= & k V_{0}^{\prime}, \\
V_{2}= & k^{2} V_{0}+l V_{0}^{\prime}, \\
= & \frac{6\left(V_{0}^{\prime \prime}+V_{0} \Psi_{0}^{\prime}\right)}{\Psi_{0}^{\prime}}+6 \Psi_{0}^{\prime}\left(\int \frac{V_{0}+\Psi_{0}^{\prime \prime \prime}}{\Psi_{0}^{\prime 2}} d Z\right) \\
& +k^{3} V_{0}^{\prime \prime \prime}+3 k l \Psi_{0}^{\prime}\left(\frac{V_{0}^{\prime}}{\Psi_{0}^{\prime}}\right) .
\end{aligned}
$$

\section{Discussion of the results}

Profiles of the non-dimensional radial and azimuthal velocity components $U$ and $V$ are shown in Figures 2(a) and 2(b) respectively for several values of the non-dimensional radial variable $\xi$, in the range $0 \leqq \xi \leqq 0.3$. The profiles were plotted by fitting together the inner solution, which is obtained by substituting the solutions for $h_{m}$ and $k_{m}(m=0,1,2,3)$ into (4.6), and the outer solution, which is obtained by substituting $\Psi_{m}$ and $V_{m}$, as calculated from (5.8) and (5.9) into (5.3). For values of $\xi$ greater than 0.3 the overlap between the inner and outer solutions is lost, as the $m \geqq 4$ terms neglected in the expansions (4.6) and (5.3) become significant. Goldstein was able to proceed further downstream than this because he was able, with the Blasius solution as the initial profile, to expand his stream function in powers of $\xi^{3 m+1}$ compared with the expansion in powers of $\xi^{m+1}$ allowed by the Cochran solution.

It is observed that the velocity profiles have adjusted rapidly to the new boundary condition in the plane $Z=0$, and are approaching smoothly towards a swirling radial jet flow before the eventual decay at large distances from the disc. As $\xi$ increases the radial velocity profile is steadily filling in and the azimuthal velocity is gradually flattening.

\section{Conclusions}

It has been shown that the matched asymptotic method used by Goldstein to solve the boundary layer equations for the near wake downstream from the trailing edge of a finite flat plate may be extended to solve the boundary equations for the near wake region of the discharge from the edge of a finite rotating disc. This is, of course, only the first step towards obtaining the complete picture of the discharge and it remains to investigate the flow in the neighbourhood of the edge of the disc and also to continue the boundary layer solution past the near wake region. Any attempt to examine the flow in the neighbourhood of the edge of the disc must necessarily involve the full Navier-Stokes equations, but the flow 


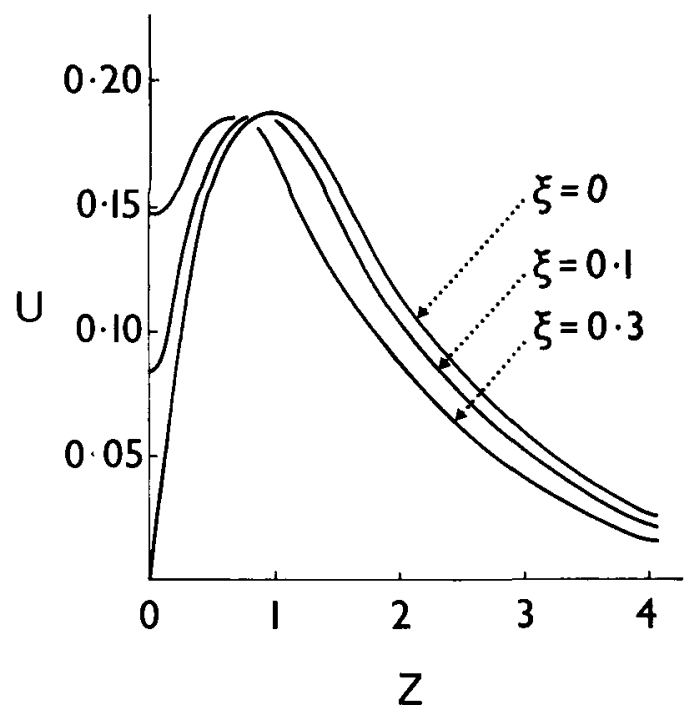

Figure 2(a)

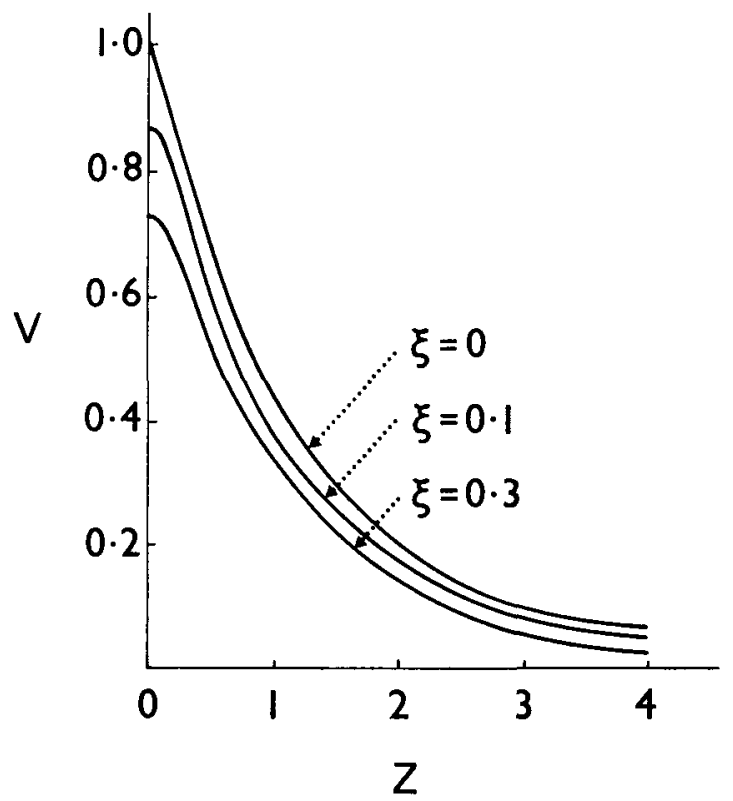

Figure (2b)

Fig. 2. Profiles of the dimensionless radial velocity, $U$, and azimuthal velocity, V, are plotted in (a) and (b) respectively for several values of $\xi$ in the range $0 \leqq \xi \leqq 0.3$. 
past the near wake region should not be difficult to obtain by using one of the many methods available for the continuation of boundary layer solutions. Goldstein's method itself may be used in a step-by-step manner to continue the boundary layer solution indefinitely but this is true in principle only and proves far too tedious in practice. A direct numerical method should prove to be most suitable.

\section{References}

[1] H. Blasius, 'Grenzschichten in Flüssigkeiten mit kleiner Reibung', Z. Math. Phys. 56 (1908), $1-37$.

[2] W. G. Cochran, 'The flow due to a rotating disc', Proc. Camb. Phil. Soc. 30 (1934), 365-375.

[3] S. Goldstein, 'Concerning some solutions of the boundary layer equations in hydrodynamics', Proc. Camb. Phil. Soc. 26 (1930), 1-30.

[4] H. Schlichting, Boundary layer theory, (Pergamon Press (1968), 93-97).

[5] R. K. Smith, 'The laminar free-convection boundary layer on a vertical heated plate in the neighbourhood of a discontinuity in the plate temperature', J. Aust. Math. Soc. (1970), $149-168$.

[6] K. Stewartson, 'On the flow near the trailing edge of a flat plate', Proc. Roy. Soc. A. 306 (1968), 275-290.

[7] K. Stewartson, 'On the flow near the trailing edge of a flat plate II', Mathematika 16 (1969), $106-121$.

[8] Th. von Karman, 'Úber laminare und turbulente Reibung', Z. angew. Math. Mech. 1 (1921), $233-252$.

Department of Mathematics

Monash University

Clayton, Victoria 